\title{
Assessment of Model Uncertainty in the Prediction of the Vibroacoustic Behavior of a Rectangular Plate by Means of Bayesian Inference
}

\author{
Nikolai Kleinfeller ${ }^{1(\otimes)}$, Christopher M. Gehb ${ }^{1}$, Maximilian Schaeffner ${ }^{1}$, \\ Christian Adams ${ }^{1}$, and Tobias Melz ${ }^{1,2}$
}

${ }^{1}$ Mechanical Engineering Department, Research Group System Reliability, Adaptive Structures and Machine Acoustics SAM, Technical University of Darmstadt, Otto-Berndt-Straße 2, 64287 Darmstadt, Germany

nikolai.kleinfeller@sam.tu-darmstadt.de

2 Fraunhofer Institute for Structural Durability and System Reliability LBF, Bartningstr. 47, 64289 Darmstadt, Germany

\begin{abstract}
Designing the vibroacoustic properties of thin-walled structures is of particularly high practical relevance in the design of vehicle structures. The vibroacoustic properties of thin-walled structures, e.g., vehicle bodies, are usually designed using finite element models. Additional development effort, e.g., experimental tests, arises if the quality of the model predictions are limited due to inherent model uncertainty. Model uncertainty of finite element models usually occurs in the modeling process due to simplifications of the geometry or boundary conditions. The latter highly affect the vibroacoustic properties of a thin-walled structure. The stiffness of the boundary condition is often assumed to be infinite or zero in the finite element model, which can lead to a discrepancy between the measured and the calculated vibroacoustic behavior. This paper compares two different boundary condition assumptions for the finite element (FE) model of a simply supported rectangular plate in their capability to predict the vibroacoustic behavior. The two different boundary conditions are of increasing complexity in assuming the stiffness. In a first step, a probabilistic model parameter calibration via Bayesian inference for the boundary conditions related parameters for the two FE models is performed. For this purpose, a test stand for simply supported rectangular plates is set up and the experimental data is obtained by measuring the vibrations of the test specimen by means of scanning laser Doppler vibrometry. In a second step, the model uncertainty of the two finite element models is identified. For this purpose, the prediction error of the vibroacoustic behavior is calculated. The prediction error describes the discrepancy between the experimental and the numerical data. Based on the distribution of the prediction error, which is determined from the results of the probabilistic model calibration, the model uncertainty is assessed and the model, which most adequately predicts the vibroacoustic behavior, is identified.
\end{abstract}

Keywords: Bayesian inference $\cdot$ Model uncertainty $\cdot$ Simply supported rectangular plates 


\section{Introduction}

The design of the vibroacoustic behavior of technical structures is of particularly high practical relevance especially in the design of vehicle structures [1]. In an early design phase, finite element (FE) models of the vehicle structures are usually built up to predict the vibroacoustic behavior, which is described in general by the natural frequencies and the associated mode shapes of the structure [1]. If the FE model predictions are limited due to inherent uncertainty, there may be additional development effort in a later design phase because the model predictions do not correspond to the real behavior of the developed structure [1]. Parameter uncertainty exists if the distributions of the model parameters are unknown [2]. Further, model uncertainty occurs if the model has been simplified, e.g., neglecting complexity of the technical structure by assuming simplified geometries or boundary conditions [2]. Boundary conditions highly affect the vibroacoustic behavior of a thin-walled structure, e.g., a rectangular plate [3]. In an experimental model, ideal boundary conditions cannot be realized. There are different ways to model the boundary conditions of the rectangular plate in the modeling process. Consequently, competing models including model uncertainty are present and the model that predicts the reality most adequately needs to be identified.

In this context, the first step is to calibrate model parameters of the competing models under consideration so that they can evolve their full prediction potential. Kennedy et al. [4] define the calibration procedure as the matching of the model predictions to observed data by identifying the unknown distributions of the model parameters. Thus, model parameter calibration adjusts the model parameters to physical observations of experimentally observed data. A deterministic model parameter calibration solves an optimization problem, e.g., [5], to achieve a best fit for the unknown model parameters based on a defined calibration criterion. Consequently, there is no information about the model parameter uncertainty after the calibration procedure. One possible way to take model parameter uncertainty into account during the calibration procedure is the application of a probabilistic model parameter calibration by means of Bayesian inference [6]. Then, the unknown model parameters are defined as random variables and their distributions are determined using the information included in the observed data. In this context, Goller et al. [6] conclude that a model never describes reality exactly. Therefore, there are no true values of the model parameters and there is always a discrepancy between the model predictions and the observed data, which is called the prediction error. Because of this fact, the model uncertainty can be assessed by evaluating the remaining prediction error after the calibration procedure [6].

This paper aims to assess two different FE models in their capability to predict the vibroacoustic behavior, i.e. the first six natural angular frequencies and mode shapes, of a simply supported rectangular plate, which is examined as an experimental model in a test stand. The two FE models differ in their modeling of the simply supported boundary conditions. A probabilistic model parameter calibration by means of Bayesian inference is performed so that the model parameter uncertainty is reduced and the FE models reach their maximum potential of predicting the vibroacoustic behavior observed in the experimental model. The model uncertainty of the two FE models is assessed based on the prediction error, which is a measure for the remaining model uncertainty after the calibration procedure. 
This paper is organized as follows. In Sect. 2, the test stand, the test specimen and the corresponding FE models are presented. In Sect. 3, a probabilistic model parameter calibration by means of Bayesian inference is introduced. Section 4 shows the results for the probabilistic model calibration for the two FE models and the model uncertainty is assessed by the prediction error. The conclusions are given in Sect. 5 .

\section{Experimental and Finite Element Models}

The data of the experimental model are obtained on a test stand of the research group SAM called SAMple test stand (System reliability, Adaptive structures, and Machine acoustics test stand for Primary Laboratory Experiments) [7]. The test stand, which is located inside a semi-anechoic room, is shown in Fig. 1(a). Here, a surrounding truss structure carries a scanning laser Doppler vibrometer (SLDV). The test specimen is an aluminium rectangular plate and is screwed to the top of an acoustic box. A detailed illustration of the test specimen with plate length $a$ and plate width $b$ is shown in Fig. 1(b). The corresponding design parameters are listed in Table 1 . The simply supported boundary conditions are realized in the experimental model according to an approach of Robin et al. [8]. Therefore, the edges of the rectangular plate are bonded to thin blades with a certain blade thickness $t_{\mathrm{bl}}$. This blades are slotted and clamped between two brackets. Thus, a defined blade length $l_{\mathrm{bl}}$ is adjusted, as illustrated in Fig. 1(c).
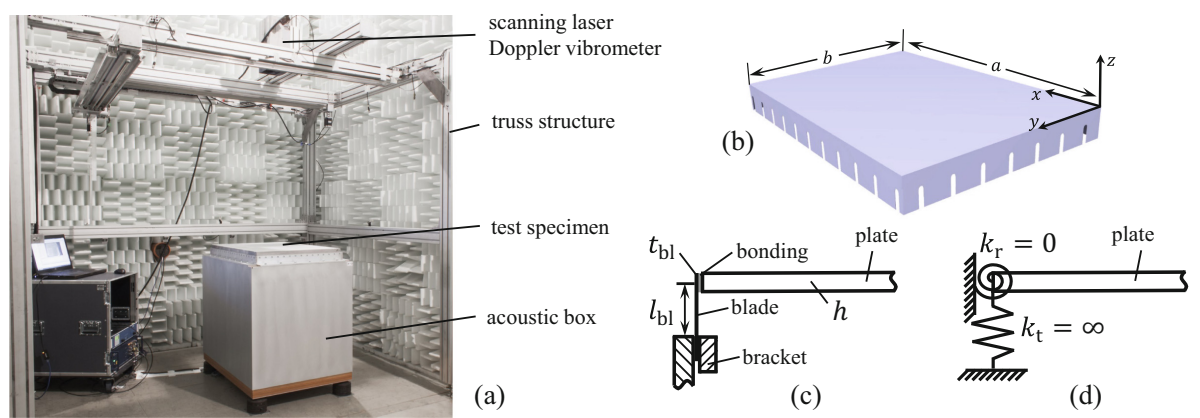

Fig. 1. (a) SAMple test stand in the semi-anechoic room [7]; (b) illustration of the simply supported rectangular plate and the corresponding design parameters; (c) mechanism of the construction to achieve simply supported boundary conditions in the experimental model; (d) model $M_{1}$ with ideal simply supported boundary condition;

The rectangular plate is excited by an automatic impact hammer with an integrated sensor for force measurement. The vibration velocities are acquired in the time domain by an SLDV measurement at defined measurement points on the surface of the test specimen. The transfer functions are determined and an experimental modal analysis is performed using a single degree-of-freedom (SDOF) analysis.

In this paper, the first six $\left(N_{\mathrm{m}}=6\right)$ natural frequencies of the rectangular plate up to $300 \mathrm{~Hz}$ are considered which are significantly involved in the low frequency excitation of the fluid inside of the acoustic box shown in Fig. 1(a). The vibrational behavior is 
Table 1: Nominal design parameters of the simply supported rectangular plate

\begin{tabular}{l|l|l|l}
\hline Parameter & Nomenclature & Value & Unit \\
\hline Plate length & $a$ & 870.0 & $\mathrm{~mm}$ \\
Plate width & $b$ & 620.0 & $\mathrm{~mm}$ \\
Plate thickness & $h$ & 5.0 & $\mathrm{~mm}$ \\
\hline Blade thickness & $t_{\mathrm{bl}}$ & 0.5 & $\mathrm{~mm}$ \\
Blade length & $l_{\mathrm{bl}}$ & 25 & $\mathrm{~mm}$ \\
\hline
\end{tabular}

measured at $N_{0}=402$ points on the surface of the plate to ensure sufficient spatial resolution. Thus, the natural angular frequencies $\omega_{r}^{(\mathrm{e})}$ and the complex mode shapes vectors $\underline{\boldsymbol{\psi}}_{r}^{(\mathrm{e})}$ with $\underline{\boldsymbol{\psi}}_{r}^{(\mathrm{e})} \in \mathbb{R}^{N_{0}}, r=1, \ldots N_{m}$ of the rectangular plate are obtained. Here, the superscript (e) $\vec{r}$ denotes the observed data of the experimental model. In order to take into account variation due to assembly in the experimental data the test specimen is reassembled and measured three times. This involves unfasten the bolts between the blades and the brackets and lifting out the rectangular plate. Then, the rectangular plate reassembled. Due to high assembly effort, a limited set $\left(N_{s}=3\right)$ of experimental data $\mathbf{D}=\left\{\omega_{1 n}^{(\mathrm{e})} \ldots \omega_{N_{\mathrm{m}} n}^{(\mathrm{e})}, \underline{\Psi}_{1 n}^{(\mathrm{e})} \ldots \underline{\Psi}_{N_{m} n}^{(\mathrm{e})}\right\}_{n=1}^{N_{s}}, n=1, \ldots, N_{s}$ is available for this paper.

In order to predict the vibroacoustic behavior of the experimental model, two competing FE models $M_{1}$ and $M_{2}$ are set up in the software ANSYS (release 19.2). For both models $M_{1}$ and $M_{2}$, the geometry of the rectanglar plate is discretized using a structuted mesh with 8-node shell elements (SHELL281) and an element size of approximately $4 \cdot 10^{3} \mathrm{~m}$. This leads to a total number of $5353 \mathrm{FE}$ nodes and to a sufficient spatial resolution for vibroacoustic analysis. The models $M_{1}$ and $M_{2}$ differ in the modeling of the simply supported boundary conditions. For model $M_{1}$, the boundary conditions of the simply supported rectangular plate are assumed by an ideal simply supporting modeling approach [3]. The nodes at the edges of the plate are directly connected to the ground. Consequently, the vertical stiffness $k_{\mathrm{t}}$ is infinite and the rotational stiffness $k_{\mathrm{r}}$ is set to zero as shown in Fig. 1(d). For model $M_{2}$, the boundary conditions of the simply supported rectangular plate are modelled by linear spring elements (COMBIN14), whose vertical stiffness $k_{\mathrm{t}}$ and rotational stiffness $k_{\mathrm{r}}$ can arbitrarily be defined. It is expected that the measured vibroacoustic behavior can be better predicted using model $M_{2}$ because it includes a more detailed modeling approach of the boundary conditions and provides two more parameter to be calibrated. Nevertheless, model $M_{1}$ is easier to implement and is used more often. An assessment of the model uncertainty should show which model is best suited to predict the bavior of the experimental model. The natural angular frequencies $\omega_{r}^{(\mathrm{n})}$ and the mode shapes $\underline{\psi}_{r}^{(\mathrm{n})}$ of both FE models $M_{1}$ and $M_{2}$ are obtained by a numerical modal analysis using a BLOCK-LANCZOS algorithm. The superscript (n) denotes the data of the numerical FE models. It is generally of interest to calibrate the unknown model parameters, which cannot be measured directly. The plate length $a$, plate width $b$ and plate thickness $h$ of the plate can be measured and, therefore assumed to be well-known. Thus, for the model $M_{1}$, the parameters of model $M_{2}$, the parameters Young's modulus $E$, mass density $\rho$ and two additional unknown model parameters the 
vertical stiffness $k_{\mathrm{t}}$ and the rotational stiffness $k_{\mathrm{r}}$ are considered in the calibration procedure. Table 2 summarizes the unknown model parameters for model $M_{1}$ and model $\mathrm{M}_{2}$.

Table 2. Unknown model parameters with lower and upper bounds

\begin{tabular}{l|l|l}
\hline Parameter & Model $M_{1}$ & Model $M_{2}$ \\
\hline$E$ & $U\left(\left[6 \cdot 10^{9}, 8 \cdot 10^{9}\right] \mathrm{Nm}^{-2}\right)$ & $U\left(\left[6 \cdot 10^{9}, 9 \cdot 10^{9}\right] \mathrm{Nm}^{-2}\right)$ \\
\hline$\rho$ & $U\left(\left[2.4 \cdot 10^{3}, 2.9 \cdot 10^{3}\right] \mathrm{kgm}^{-3}\right)$ & $U\left(\left[2.4 \cdot 10^{3}, 2.9 \cdot 10^{3}\right] \mathrm{kgm}^{-3}\right)$ \\
\hline$k_{\mathrm{t}}$ & --- & $U\left(\left[10^{4}, 10^{7}\right] \mathrm{Nm}^{-1}\right)$ \\
\hline$k_{\mathrm{r}}$ & --- & $U\left(\left[0,10^{2}\right] \mathrm{Nm}\right)$ \\
\hline
\end{tabular}

It is further assumed that no prior knowledge about the distribution of the unknown model parameters is available. Consequently, the unknown model parameters are assumed to be uniformly distributed $(U)$ between the lower and upper bounds, which are given in Table 2. The respective lower and upper bounds of the model parameters are best guesses so that the calibration is not restricted. A uniform distribution is a common choice if no information about the model parameters is available [9].

The introduced experimental model and the FE models are embedded within a probabilistic model parameter calibration by means of Bayesian inference, which is described in the following section. Within this framework, the unknown model parameters to be calibrated are defined as random variables and are combined in the vector of unknown parameters.

$$
\boldsymbol{\theta}=\left\{\begin{array}{c}
(E, \rho) \text { for } M_{1}, \\
\left(E, \rho, k_{\mathrm{t}}, k_{\mathrm{r}}\right) \text { for } M_{2}
\end{array}\right.
$$

\section{Probabilistic Parameter Calibration by Means of Bayesian Inference}

The aim of a probabilistic parameter calibration is the identification of the unknown distributions of the model parameters. The Bayesian Theorem [9] describes the posterior probability density as

$$
p\left(\boldsymbol{\theta} \mid \mathbf{D}, M_{i}\right)=c^{-1} p\left(\mathbf{D} \mid \boldsymbol{\theta}, M_{i}\right) p\left(\boldsymbol{\theta} \mid M_{i}\right) .
$$

The prior probability density $p\left(\boldsymbol{\theta} \mid M_{i}\right)$ quantifies the prior probability of a specific parameter set $\boldsymbol{\theta}$ of Eq. (2) for a model $M_{i}$. The likelihood $p\left(\mathbf{D} \mid \boldsymbol{\theta}, M_{i}\right)$ quantifies the probability that a specific model evaluation of the model $M_{i}$ with the parameter set $\boldsymbol{\theta}$ corresponds 
to the experimental model data $\mathbf{D}$. The parameter $c$, the total probability or evidence, is typically not computable with reasonable effort and is only normalizing the result anyway [10]. To avoid this effort, methods for sampling Eq. (2) can be chosen. In this paper, a Transitional Markov Chain Monte Carlo (TMCMC) algorithm by Ching et al. [11] is used to solve Eq. (2). In the context of TMCMC, Eq. (2) is not solved directly, but it is assumed that the posterior probability

$$
p_{j} \propto p\left(\mathbf{D} \mid \boldsymbol{\theta}, M_{i}\right)^{q_{j}} p\left(\boldsymbol{\theta} \mid M_{i}\right)
$$

of a calculation step $j=0, \ldots, m$ is proportional to the product of the prior probability $p\left(\boldsymbol{\theta} \mid M_{i}\right)$ and the likelihood $p(\mathbf{D} \mid \boldsymbol{\theta}, M)^{q_{j}}$.Thus, the problem is solved using $m>1$ calculation steps. The power $q_{j}$ is defined as

$$
q_{j} \in[0,1], q_{0}=0<q_{1}<\ldots<q_{m}=1
$$

and is used to control the transition of the prior distribution to the posterior distribution. Thus, the samples are gradually moved from the prior $\left(j=0, q_{0}=0\right)$ to the posterior distribution $\left(j=m, q_{m}=1\right)$. During each step, the parameter space is resampled with a certain number of $N_{\mathrm{s}}$ points. The TMCMC terminates with the condition $q_{m}=1$. The TMCMC overcomes the weaknesses of methods, which are working with Markov Chain Monte Carlo (MCMC), e. g., the reduced statistical efficiency, the presence of the burn-in period, and the inefficiency of the calibration procedure for a high number of model parameters [12]. More detailed information can be found in [11, 12].

The likelihood $p\left(\mathbf{D} \mid \boldsymbol{\theta}, M_{i}\right)$ includes the discrepancy between the FE model outputs and the experimental data, which is termed the prediction error [6, 13]. A formulation for the likelihood $p\left(\mathbf{D} \mid \boldsymbol{\theta}, M_{i}\right)$ based on modal properties is proposed by Vanik et al. [14] and is also adopted by Goller et al. [6]. It is assumed based on the principle of maximum entropy that the prediction error follows a normal distribution [15]. Additionally to modal properties the likelihood used for this contribution also contains the mass $m$ of the rectangular plate as a model output. The likelihood of the data set $\mathbf{D}$ for a model parameter set $\boldsymbol{\theta}$ and a model $M_{i}$

$$
p\left(\mathbf{D} \mid \boldsymbol{\theta}, M_{i}\right)=c_{1} \exp \left(-\frac{1}{2} \sum_{n=1}^{N_{s}} \sum_{r=1}^{N_{m}}\left(\varepsilon_{r}^{-2} e_{\omega_{r n}^{2}}^{2}+\delta_{r}^{-2} e_{\psi, r n}^{2}\right)+\sigma^{-2} e_{\text {mass }}^{2}\right)
$$

is then composed using the prediction error of the natural angular frequencies

$$
e_{\omega_{r n}^{2}}=\omega_{r n}^{2(\mathrm{e})}-\omega_{r}^{2(\mathrm{n})},
$$

the prediction error of the mode shapes

$$
e_{\psi, r n}=1-\frac{\left|\underline{\boldsymbol{\Psi}}_{r n}^{(\mathrm{e}) \mathrm{T}} \underline{\boldsymbol{\Psi}}_{r}^{(\mathrm{n})}\right|^{2}}{\underline{\boldsymbol{\Psi}}_{r}^{(\mathrm{n}) \mathrm{T}} \underline{\boldsymbol{\Psi}}_{r}^{(\mathrm{n})}}
$$

and the prediction error of the mass.

$$
e_{\mathrm{mass}}=\frac{m^{(\mathrm{e})}}{m^{(\mathrm{n})}}-1
$$


The factor $c_{1}$ is described in detail in [14] and just normalizes the likelihood. The prediction error variances of the natural angular frequencies $\varepsilon_{r}^{2}$ and of the mode shapes $\delta_{r}^{2}$ are estimated from the three observed data sets. Consequently, individual values of $\varepsilon_{r}^{2}$ and $\delta_{r}^{2}$ are used for the first six mode shapes. It is assumed that the prediction error variance of the mass has a constant value of $\sigma^{2}=10^{-2}$. The prediction error of the mode shapes $e_{\psi, r n}$ in Eq. (7) corresponds to the modal assurance criterion (MAC). In vibroacoustics, the agreement of the numerically and experimentally obtained mode shapes is usually assessed by the MAC [16]. The MAC describes the linear correlation between the vector of the mode shapes of the experimental model $\underline{\Psi}_{r}^{(\mathrm{e})}$ and those of the numerical model $\underline{\psi}_{r}^{(\mathrm{n})}$. In Eq. (7), the vector of the mode shapes for the experimental $\underline{\Psi}_{r}^{(\mathrm{e})}$ and for the numerical model $\underline{\Psi}_{r}^{(\mathrm{n})}$ must have equal length and take into account equal spatial coordinates. To match the number of points of the experimental model, only the closest node of the FE mesh in relation to a measurement point is considered. Thus, the vector of the mode shapes of the FE model is reduced from 5353 to 402 points. The results of the probabilistic model parameter calibration are shown in the following section.

\section{Calibration Results and Assessment of the Model Uncertainty}

A probabilistic model parameter calibration by means of TMCMC is performed for both models $M_{1}$ and $M_{2}$, whereby a number of $N=1000$ is used to cover the parameter space. The computations are performed on a standard desktop PC with a single model evaluation taking approximately $20 \mathrm{~s}$. In this paper, only the results for the natural angular frequencies are shown and discussed in detail since the results of the mode shapes lead to similar conclusions. Nevertheless, the natural angular frequencies as wells as the mode shapes are used to compute the likelihood following Eq. (5).

Figure 2 depicts the posterior distributions of the calibrated model parameters Young's modulus $E$ (a) and mass density $\rho$ (b) for model $M_{1}$ as histogramms.
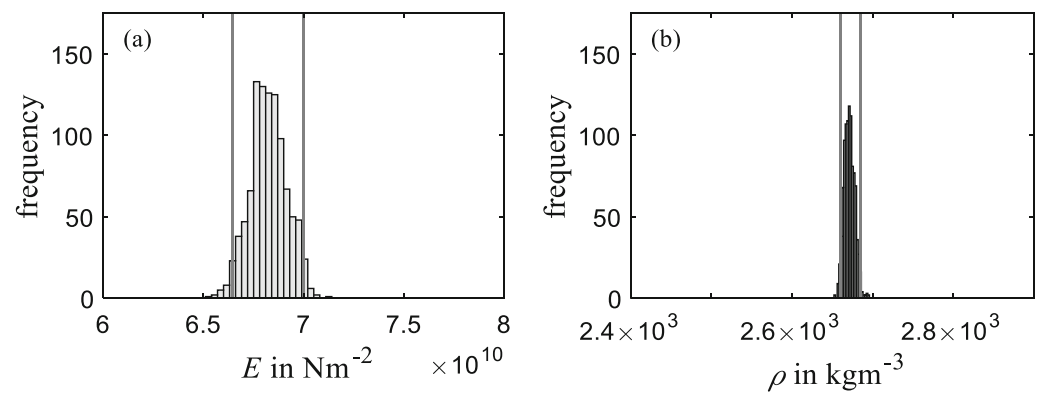

Fig. 2. Posterior distributions with the $95 \%$ interpercentile intervals (solid lines) of the calibrated model parameters Young's modulus $E$ (a) and mass density $\rho$ (b) for model $M_{1}$. The limits of the abscissae correspond to the lower and upper bounds of the prior distributions of the unknown model parameters. 
As expected, the distributions of the model parameters $E$ and $\rho$ are narrowed down by the information contained in the observed data set $\mathbf{D}$. The lower and upper bounds of the prior distributions are represented by the limits of the abscissa in Fig. 2. The relations between the limits of the abscissae and the $95 \%$ interpercentile intervalls of the posterior distributions show the reduction of model parameter uncertainty due to the probablistic model calibration. The model parameter ranges are reduced by approximetly $82 \%$ for the Young's modulus $E$ and $95 \%$ for the mass density $\rho$.

The effect of the probabilistic model parameter calibration procedure on the accuracy of the model prediction of the model $M_{1}$ is shown in Fig. 3. Here, the histograms of the prior and posterior distributions of the first six $\left(N_{\mathrm{m}}=6\right)$ natural angular frequencies $\omega_{r}^{(\mathrm{n})}$, the $95 \%$ interpercentile intervals of the posterior distribution as well as the corresponding observed data $\mathbf{D}=\left\{\omega_{1}^{(\mathrm{e})} \ldots \omega_{N_{\mathrm{m}} n}^{(\mathrm{e})}\right\}_{n=1}^{N}, N=3$, are plotted. As expected, the distributions of the posterior model predictions using calibrated model parameters are narrowed down in comparison to the distribution of the prior model predictions using non-calibrated model parameters. For the second to the sixth natural angular frequency (b)-(f), the posterior model predictions are closely distributed around the observed data values.
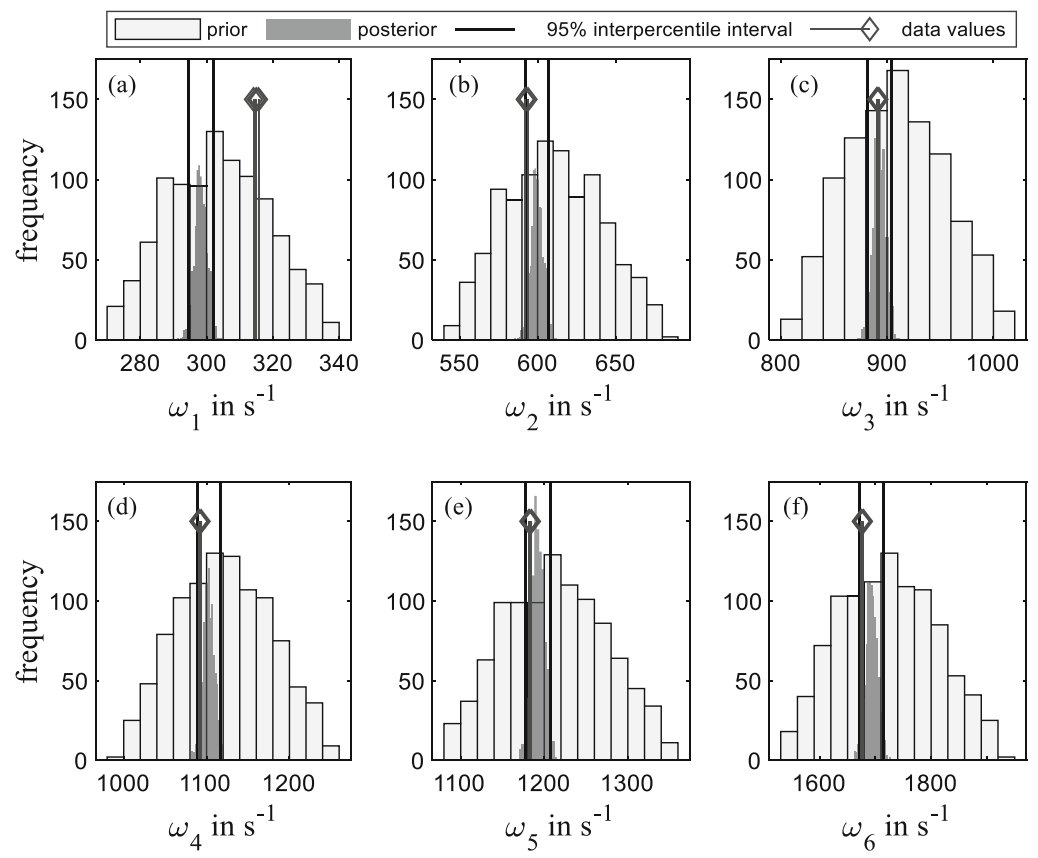

Fig. 3. Distributions of the first six natural angular frequencies of the simply supported rectangular plate predicted with the model $M_{1}$ using non-calibrated (prior) and calibrated (posterior) model parameters. The $95 \%$ interpercentile intervals of the posterior distributions are plotted as solid lines. The corresponding values of the observed data are plotted as stems. 
In the case of the first natural angular frequency $\omega_{1}$ (a), a deviation between the observed data values and the posterior model predictions still remains, which can not be further reduced by the calibration procedure. This leads to the fact, that the observed data values are not part of the posterior distribution of the model predition of the first natural angular frequency $\omega_{1}$.

Figure 4 depicts the posterior distributions of the calibrated model parameters Young's modulus $E(\mathrm{a})$, mass density $\rho(\mathrm{b})$, vertical stiffness $\mathrm{k}_{\mathrm{t}}(\mathrm{c})$, and rotational stiffness $k_{\mathrm{r}}(\mathrm{d})$ for model $M_{2}$ as histogramms. The distributions of the model parameters Young's modulus $E$, mass density $\rho$ and rotational stiffness $k_{\mathrm{r}}$ are also narrowed down by the information contained in the observed data sets $\mathbf{D}$. The vertical stiffness $k_{\mathrm{t}}$ is still distributed over the entire range even after the calibration. The data $\mathbf{D}$ do not contain the necessary information to extend the knowledge of the vertical stiffness $k_{\mathrm{t}}$ due to the calibration procedure. The lower and upper bounds of the prior distributions are represented by the limits of the abscissae in Fig. 4. The relations between the limits of the abscissae and the $95 \%$ interpercentile intervalls of the posterior distributions visualize the reduction of model parameter uncertainty for the model $M_{2}$ due to the probablistic model calibration. The model parameter ranges are reduced by approximetly $71 \%$ for the Young's modulus $E, 94 \%$ for the mass density $\rho$ and $84 \%$ for the rotational stiffness $k_{\mathrm{r}}$.
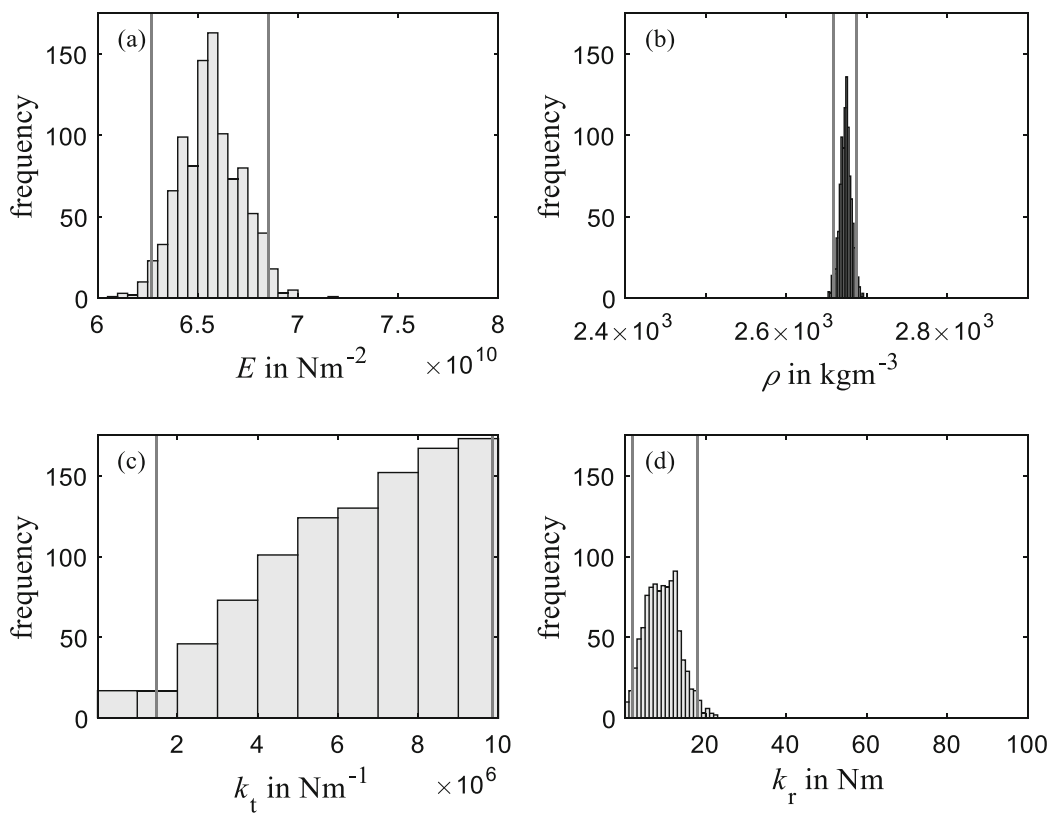

Fig. 4. Posterior distributions with the $95 \%$ interpercentile intervals (solid lines) of the calibrated model parameters Young's modulus $E$ (a), mass density $\rho$ (b), vertical stiffness $k_{\mathrm{t}}$ (c) and rotational stiffness $k_{\mathrm{r}}(\mathrm{d})$ for model $M_{2}$. The limits of the abscissae correspond to the lower and upper bounds of the prior distributions of the unknown model parameters. 
For the vertical stiffness $\mathrm{k}_{\mathrm{t}}$, a reduction of the model parameter ranges cannot be achieved by the probabilistic model parameter calibration procedure.

The effect of the probabilistic model parameter calibration procedure on the accuracy of the model prediction of the model $M_{2}$ is shown by Fig. 5. Here, the histograms of the prior and posterior distributions of the first six $\left(N_{\mathrm{m}}=6\right)$ natural angular frequencies $\omega_{r}^{(\mathrm{n})}$, the $95 \%$ interpercentile intervals of the posterior distribution as well as the corresponding observed data $\mathbf{D}=\left\{\omega_{1}^{(\mathrm{e})} \ldots \omega_{N_{\mathrm{m}} n}^{(\mathrm{e})}\right\}_{n=1}^{N}, N=3$, are plotted. The distributions of the posterior model predictions using the calibrated model parameters are narrowed down in comparison to the distribution of the prior model predictions using non-calibrated model parameters. For the first to the sixth natural angular frequency see Fig. 5(a)-(f), the posterior model predictions are closely distributed around the observed data values. Consequently, the observed data values are always part of the posterior distribution of the model preditions of the first six natural angular frequencies $\omega_{r}^{(\mathrm{n})}$. It can be concluded that the preditction quality of the models $M_{1}$ and $M_{2}$ has improved due to the probabilistic model parameter calibration procedure.

Table 3 summarizes the results of the calibrated model parameters for the models $M_{1}$ and $M_{2}$ by means of the $95 \%$ interpercentiles and the mean value of the distributions.

Table 3. Posterior uncertainty of the calibrated model parameters for the model $M_{1}$ and $M_{2}$

\begin{tabular}{|c|c|c|c|c|c|c|}
\hline \multirow{3}{*}{ Parameter } & \multicolumn{3}{|l|}{ Model $M_{1}$} & \multicolumn{3}{|l|}{ Model $M_{2}$} \\
\hline & \multicolumn{2}{|c|}{$95 \%$ interpercentile } & \multirow[t]{2}{*}{ mean } & \multicolumn{2}{|c|}{$95 \%$ interpercentile } & \multirow[t]{2}{*}{ mean } \\
\hline & $\min$ & $\max$ & & $\min$ & $\max$ & \\
\hline$E$ in $\mathrm{Nm}^{-2}$ & $6.64 \cdot 10^{10}$ & $7.00 \cdot 10^{10}$ & $6.82 \cdot 10^{10}$ & $6.27 \cdot 10^{10}$ & $6.85 \cdot 10^{10}$ & $6.56 \cdot 10^{10}$ \\
\hline$\rho$ in $\mathrm{kgm}^{-3}$ & $2.66 \cdot 10^{3}$ & $2.68 \cdot 10^{3}$ & $2.67 \cdot 10^{3}$ & $2.66 \cdot 10^{3}$ & $2.69 \cdot 10^{3}$ & $2.67 \cdot 10^{3}$ \\
\hline$k_{\mathrm{t}}$ in $\mathrm{Nm}^{-1}$ & --- & --- & --- & $1.47 \cdot 10^{6}$ & $9.84 \cdot 10^{6}$ & $6.58 \cdot 10^{6}$ \\
\hline$k_{\mathrm{r}}$ in $\mathrm{Nm}$ & -- & --- & -- & 1.94 & 18 & 9.3 \\
\hline
\end{tabular}

Finally, the model uncertainty of the models $M_{1}$ and $M_{2}$ is assessed based on the prediction error of the first six natural angular frequencies of the rectangluar plate. Goller et al. [6] conclude in their contribution that the distribution of the prediction error after the calibration procedure is a measure for the remaining model uncertainty, which can not be further reduced by adjusting the model parameter.

In Fig. 6 the distributions of the prediction error of the natural angular frequencies $e_{\omega_{r}^{2}}$ averaged over all three observed data values are plotted for the first six natural angular frequencies $\left(N_{\mathrm{m}}=6\right)$ and for the models $M_{1}$ and $M_{2}$, respectively. The prediction error $e_{\omega_{r}^{2}}$ is calculated using Eq. (6) and the model predictions are based on calibrated model parameters. If the model predictions of the natural angular frequencies $\omega_{r}^{(\mathrm{n})}$ match the observed data values on average, the prediction error $e_{\omega_{r}^{2}}$ is distributed around zero and consequently, the averaged prediction error $\bar{e}_{\omega_{r}^{2}}=0$. Table 4 shows the averaged prediction errors $\bar{e}_{\omega_{r}^{2}}$ of the first six natural angular frequencies for model $M_{1}$ and model $M_{2}$. The averaged prediction errors $\bar{e}_{\omega_{r}^{2}}$ are plotted as stems in Fig. 6. 

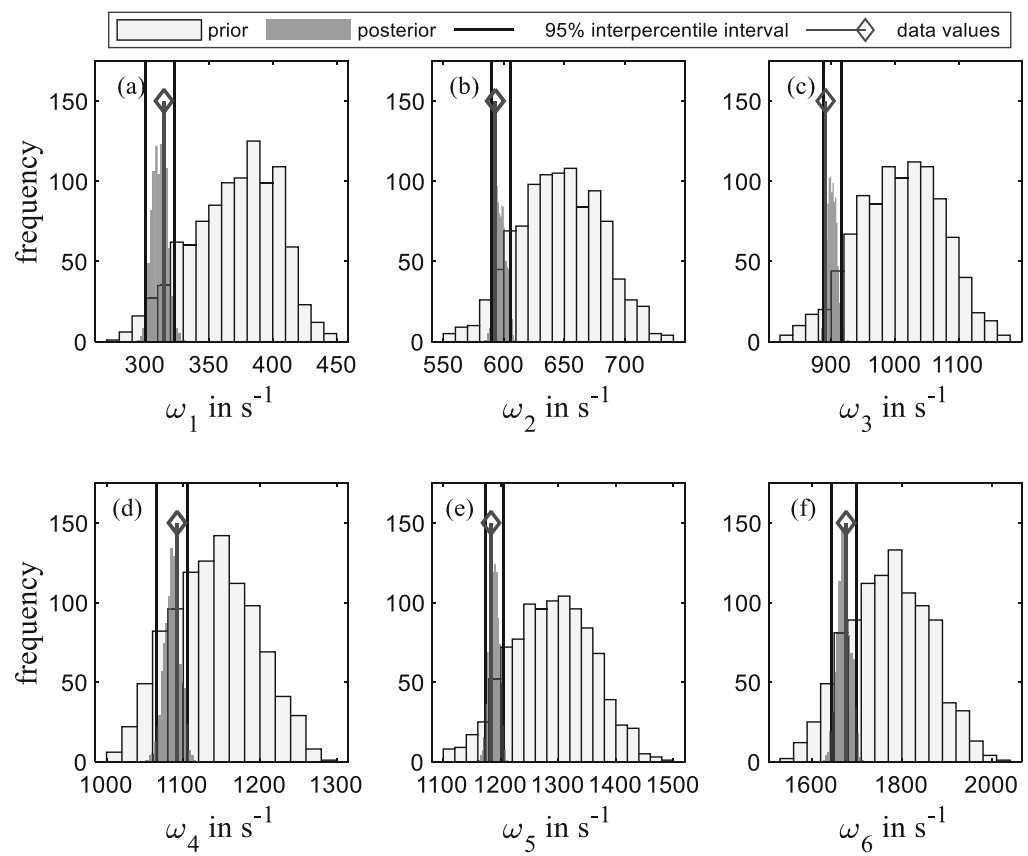

Fig. 5. Distributions of the first six natural angular frequencies of the simply supported rectangular plate predicted with the model $M_{2}$ using non-calibrated (prior) and calibrated (posterior) model parameters. The $95 \%$ interpercentile intervals of the posterior distributions are plotted as solid lines. The corresponding values of the observed data are plotted as stems.

Table 4. Averaged prediction error $\bar{e}_{\omega_{r}^{2}}$ in $\mathrm{s}^{-2}$ of the first six natural angular frequencies $\omega_{r}^{(n)}$ of the simply supported rectangular plate for the models $M_{1}$ and $M_{2}$

\begin{tabular}{l|l|l|l|l|l|l}
\hline & $\bar{e}_{\omega_{1}^{2}}$ & $\bar{e}_{\omega_{2}^{2}}$ & $\bar{e}_{\omega_{3}^{2}}$ & $\bar{e}_{\omega_{4}^{2}}$ & $\bar{e}_{\omega_{5}^{2}}$ & $\bar{e}_{\omega_{6}^{2}}$ \\
\hline$M_{1}$ & $-1.017 \cdot 10^{4}$ & $0.828 \cdot 10^{4}$ & $0.307 \cdot 10^{4}$ & $2.401 \cdot 10^{4}$ & $2.355 \cdot 10^{4}$ & $5.952 \cdot 10^{4}$ \\
\hline$M_{2}$ & $-0.205 \cdot 10^{4}$ & $0.554 \cdot 10^{4}$ & $1.835 \cdot 10^{4}$ & $-1.356 \cdot 10^{4}$ & $1.211 \cdot 10^{4}$ & $-1.427 \cdot 10^{4}$ \\
\hline
\end{tabular}

It can be concluded that except for the third natural angular frequency $\omega_{3}^{(n)}$, the model $M_{2}$ has a smaller averaged prediction errors $\bar{e}_{\omega_{r}^{2}}$ than the model $M_{1}$. Consequently, the model $M_{2}$ leads to a better prediction of the vibroacoustic behavior of the simply supported rectangluar plate for the first six natural angular frequencies. A possible option to close the remaining shift of the prediction error is the definition and calibration of a discrepancy function according to Kennedy and O'Hagan [4]. This can be done by a Gaussian process as shown by Feldmann et al. [17]. 

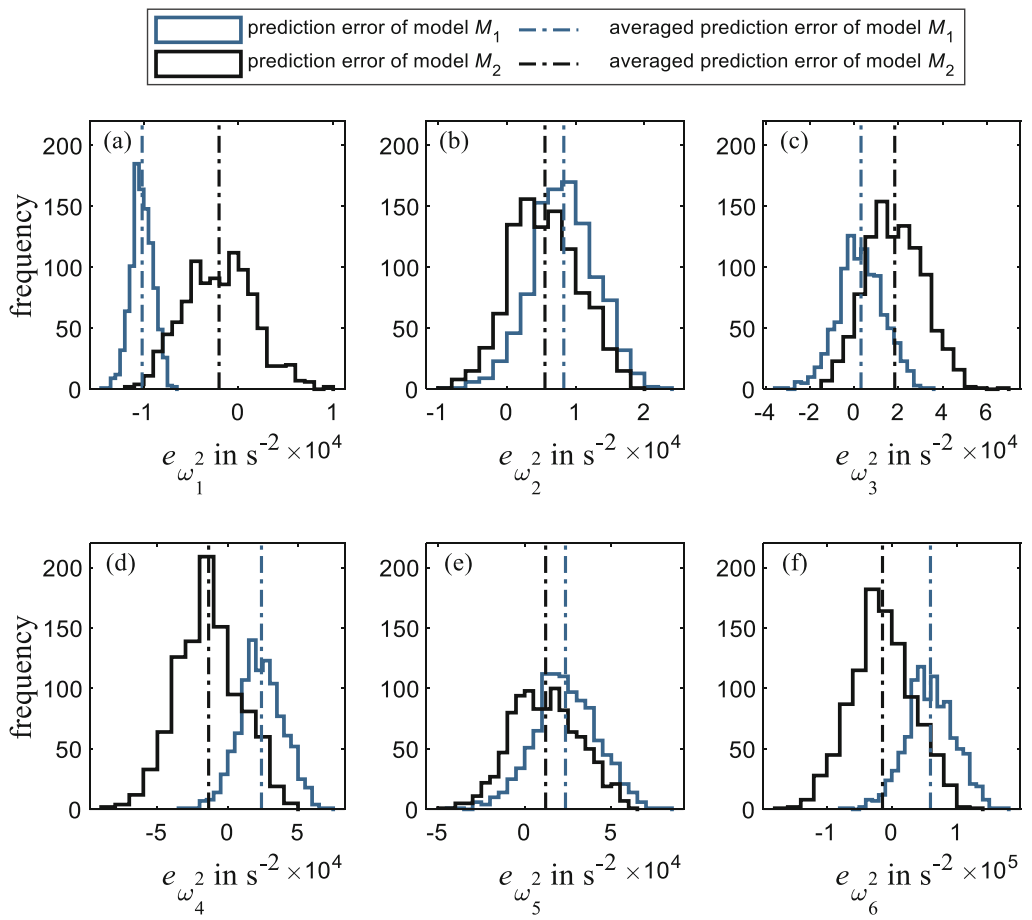

Fig. 6. Distributions of the prediction error $e_{\omega_{r}^{2}}$ after the calibration procedure for the first six natural angular frequencies of the simply supported rectangular plate using model $M_{1}$ and model $M_{2}$ respectively. The averaged prediction errors $\bar{e}_{\omega_{r}^{2}}$ are plotted as stems.

\section{Conclusions}

This paper presents the assessment of the model uncertainty in the prediction of the vibroacoustic behavior of a simply supported rectangular plate. For this purpose, a probabilistic model calibration via Bayesian inference is performed and the model uncertainty is assessed by the remaining prediction error after the calibration procedure. The test specimen is a simply supported rectangular plate which is investigated at a test stand. The rectangular plate is excited by an automatic impact hammer and the vibration velocities are obtained by a SLDV at specific measurement points on the surface of the plate. The vibroacoustic behavior is described by the natural angular frequencies and the corresponding mode shapes of the simply supported rectangular plate. Consequently, the vibroacoustic behavior of the experimental model is obtained by an experimental modal analysis using the measured transfer functions. Two competing FE models for the prediction of the vibroacoustic behavior of the simply supported rectangular plate are set up, which differ in the modeling of the simply supported boundary conditions. Both models are embedded in a probabilistic model calibration procedure via Bayesian inference.

The aim of the calibration is the reduction of the model parameter uncertainty by the identification of the distribution of the unknown model parameters. The measurement data used for the calibration are the results of the experimental modal analysis. The 
likelihood for the Bayesian inference consists of three parts, which consider the natural angular frequencies, the corresponding mode shapes as well as the mass of the simply supported rectangular plate. For both FE models, a significant reduction of the lower and upper bounds of the unknown model parameters is achieved by means of probabilistic model calibration. It is concluded that the model parameter uncertainty is reduced for both models. For assessment of the model uncertainty involved in the FE models, the remaining prediction error, which is a measure for the model uncertainty, is analyzed after the calibration. It is shown that a more detailed modeling of the boundary conditions leads to better calibration results. For future work, the experimental data base has to be extended for the calibration procedure in order to better take into account the scattering of the vibroacoustic behavior occurring in reality due to the assembling process and to validate the prediction of the vibroacoustic behavior against independent measurement data.

Acknowledgements. The authors like to thank the Deutsche Forschungsgemeinschaft (DFG, German Research Foundation) for funding this project within the Sonderforschungsbereich (SFB, Collaborative Research Center) 805 "Control of Uncertainties in Load-Carrying Structures in Mechanical Engineering" - project number: 57157498. The authors also acknowledge the financial support for the 3D scanning laser Doppler vibrometer from the DFG under the grant number INST $163 / 520-1$.

\section{References}

1. Luegmair, M., Schmid, J.D.: Challenges in vibroacoustic vehicle body simulation including uncertainties. SAE Technical Paper 2020-01-1571 (2020)

2. Mallapur, S., Platz, R.: Uncertainty quantification in the mathematical modelling of a suspension strut using Bayesian inference. Mech. Syst. Signal Proc. 118, 158-170 (2019)

3. Bapat, A.V., Suryanarayan, N.: A theoretical basis for the experimental realization of boundary conditions in the vibration analysis of plates. J. Sound Vibr. 163(3), 463-478 (1993)

4. Kennedy, M.C., O’Hagan, A.: Bayesian calibration of computer models. J. Roy. Stat. Soc. Ser. B (Stat. Methodol.) 63(3), 425-464 (2001)

5. Wang, X., Lin, S.I., Wang, S.: Dynamic friction parameter identification method with LuGre model for direct-drive rotary torque motor. Math. Probl. Eng. 2016, 1-8 (2016)

6. Goller, B., Schuëller, B.I.: Investigation of model uncertainties in Bayesian structural model updating. J. Sound Vibr. 330, 6122-6136 (2011)

7. Adams, C., Bös, J., Slomski, E.M., Melz, T.: An experimental investigation of vibrating plates in similitude and the possibility to replicate the responses using sensitivity-based scaling laws. In: 6th Conference on Noise and Vibration emerging methods, Ibiza, pp. 175574-1-175574-12 (2018)

8. Robin, O., Chazot, A.D., Boulandet, R., Michau, M., Berry, A., Atalla, N.: A plane and thin panel with representative simply supported boundary conditions for laboratory vibroacoustic tests. Acta Acustica United with Acustica 102(1), 403-425 (2016)

9. Smith, R.C.: Uncertainty Quantification: Theory, implementation and application Computational Science \& Engineering, 12th edn. Society for Industrial and Applied Mathematics, Philadelphia (2014)

10. Green, P.L., Worden, K.: Modelling friction in a nonlinear dynamic system via Bayesian inference. In: Allemang, R., et al. (eds.) Special Topics in Structural Dynamics, Vol. 6, pp. 543 553. Springer, New York (2013) 
11. Ching, J., Chen, Y.-C.: Transitional Markov Chain Monte Carlo method for Bayesian model updating, model class selection, and model averaging. J. Eng. Mech. 133(7), 816-832 (2007)

12. Betz, W., Papaioannou, I., Straub, D.: Transitional Markov Chain Monte Carlo: observations and improvements. J. Eng. Mech. 142(5), 04016016 (2016)

13. Prajapat, K., Ray-Chaudhuri, S.: Prediction error variances in Bayesian model updating employing data sensitivity. J. Eng. Mech. 142(12), 04016096 (2016)

14. Vanik, M., Beck, J., Au, S.-K.: Bayesian probabilistic approach to structural health monitoring. J. Eng. Mech. 126, 738-745 (2000)

15. Jaynes, E.T.: Probability Theory: The logic of science. Cambridge University Press, Cambridge (2003)

16. Allemang, R.J.: The modal assurance criterion - twenty years of use and abuse. Sound Vibr. 1(August), 14-21 (2003)

17. Feldmann, R., Platz, R.: Assessing model form uncertainty for a suspension strut using Gaussian Processes. In: 3rd ECCOMAS Thematic Conference on Uncertainty Quantification in Computational Sciences and Engineering, Crete, pp. 569-582 (2019)

Open Access This chapter is licensed under the terms of the Creative Commons Attribution 4.0 International License (http://creativecommons.org/licenses/by/4.0/), which permits use, sharing, adaptation, distribution and reproduction in any medium or format, as long as you give appropriate credit to the original author(s) and the source, provide a link to the Creative Commons license and indicate if changes were made.

The images or other third party material in this chapter are included in the chapter's Creative Commons license, unless indicated otherwise in a credit line to the material. If material is not included in the chapter's Creative Commons license and your intended use is not permitted by statutory regulation or exceeds the permitted use, you will need to obtain permission directly from the copyright holder.

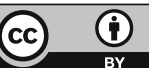

